\title{
HIFI spectroscopy of low-level water transitions in M 82^
}

\author{
A. Weiß $\beta^{1}$, M. A. Requena-Torres ${ }^{1}$, R. Güsten ${ }^{1}$, S. García-Burillo ${ }^{2}$, A. I. Harris ${ }^{3}$, F. P. Israel ${ }^{4}$, T. Klein ${ }^{1}$, C. Kramer ${ }^{5}$, \\ S. Lord ${ }^{6}$, J. Martin-Pintado ${ }^{7}$, M. Röllig ${ }^{8}$, J. Stutzki ${ }^{8}$, R. Szczerba ${ }^{9}$, P. P. van der Werf ${ }^{4}$, S. Philipp-May ${ }^{1}$, H. Yorke ${ }^{10}$, \\ M. Akyilmaz ${ }^{8}$, C. Gal ${ }^{8}$, R. Higgins ${ }^{13}$, A. Marston ${ }^{12}$, J. Roberts ${ }^{7}$, F. Schlöder ${ }^{8}$, M. Schultz ${ }^{8}$, D. Teyssier ${ }^{12}$, \\ N. Whyborn ${ }^{11}$, and H. J. Wunsch ${ }^{1}$
}

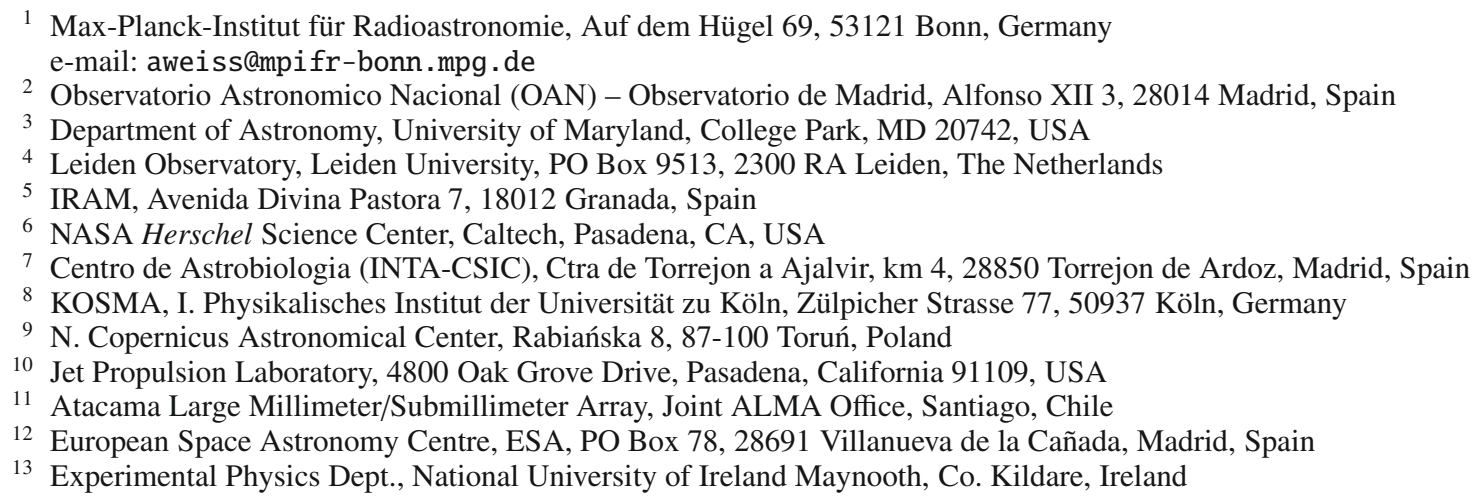

Received 28 May 2010 / Accepted 1 July 2010

\section{ABSTRACT}

\begin{abstract}
We present observations of the rotational ortho-water ground transition, the two lowest para-water transitions, and the ground transition of ionised ortho-water in the archetypal starburst galaxy M 82, performed with the HIFI instrument on the Herschel Space Observatory. These observations are the first detections of the para- $\mathrm{H}_{2} \mathrm{O}\left(1_{11}-0_{00}\right)(1113 \mathrm{GHz})$ and ortho- $\mathrm{H}_{2} \mathrm{O}^{+}\left(1_{11}-0_{00}\right)(1115 \mathrm{GHz})$ lines in an extragalactic source. All three water lines show different spectral line profiles, underlining the need for high spectral resolution in interpreting line formation processes. Using the line shape of the para- $\mathrm{H}_{2} \mathrm{O}\left(1_{11}-0_{00}\right)$ and ortho- $\mathrm{H}_{2} \mathrm{O}^{+}\left(1_{11}-0_{00}\right)$ absorption profile in conjunction with high spatial resolution $\mathrm{CO}$ observations, we show that the (ionised) water absorption arises from a $\sim 2000 \mathrm{pc}^{2}$ region within the HIFI beam located about $\sim 50 \mathrm{pc}$ east of the dynamical centre of the galaxy. This region does not coincide with any of the known line emission peaks that have been identified in other molecular tracers, with the exception of HCO. Our data suggest that water and ionised water within this region have high (up to 75\%) area-covering factors of the underlying continuum. This indicates that water is not associated with small, dense cores within the ISM of M 82 but arises from a more widespread diffuse gas component.
\end{abstract}

Key words. line: formation - galaxies: ISM - ISM: molecules - galaxies: individual: M 82 - infrared: galaxies submillimeter: galaxies

\section{Introduction}

High-resolution spectroscopy of far-infrared and sub-millimetre water lines is an important tool for studying the physical and chemical properties of the interstellar medium (ISM). Absorption by terrestrial atmospheric water vapour has groundbased studies of water in extragalactic systems limited to radio maser transitions (such as the famous $22 \mathrm{GHz}$ water line) or to a few systems with significant redshift (e.g. Combes \& Wiklind 1997; Cernicharo et al. 2006; Menten et al. 2008). Earlier satellite missions, such as Odin and SWAS, did not have enough collecting area to detect the relatively faint ground transitions of

* Herschel is an ESA space observatory with science instruments provided by European-led Principal Investigator consortia and with participation from NASA. water in external galaxies. ISO and, more recently, Spitzer have provided the first systematic studies of water in the far infrared (IR) regime (e.g. Fischer et al. 1999; Gonzalez-Alfonso 2004). These missions, however, did not cover the frequencies of the water ground transitions and other low-level water lines.

Only with the launch of the Herschel satellite, with its large collecting area, have these transitions become accessible in the nearby universe (e.g. van der Werf et al. 2010). As part of the HEXGAL guaranteed time key program (PI Güsten), we are surveying the low-level water lines in different nuclear environments, performing velocity-resolved spectroscopy with the Heterodyne Instrument for the Far Infrared (HIFI, de Graauw et al. 2010). In this letter we report on our first observations towards the central region of the archetypal starburst galaxy M 82. We adopt a distance of 3.9 Mpc (Sakai \& Madore 1999). 


\section{Observations and data reduction}

Using the HIFI instrument onboard Herschel, we have observed the ground transitions of ortho and para water, o- $\mathrm{H}_{2} \mathrm{O}\left(1_{10}-1_{01}\right)$ and $\mathrm{p}-\mathrm{H}_{2} \mathrm{O}\left(1_{11}-0_{00}\right)$, as well as the $\mathrm{p}-\mathrm{H}_{2} \mathrm{O}\left(2_{02}-1_{11}\right)$ line, towards the centre of M $82\left(\mathrm{RA}=09^{\mathrm{h}} 55^{\mathrm{m}} 52^{\mathrm{s}} .22 \mathrm{Dec}=69^{\circ} 40^{\prime} 46^{\prime \prime} .9\right.$ $\mathrm{J} 2000$ ). Observations were carried out in fast-chopping dualbeamswitch mode using a wobbler throw of $3^{\prime}$ for all observations. The wobbler frequency was $0.8,1.4$, and $2.0 \mathrm{~Hz}$ for the $557 \mathrm{GHz}, 988 \mathrm{GHz}$, and $1113 \mathrm{GHz}$ observations, respectively. Nodding was performed every $\sim 40 \mathrm{~s}$. Calibration was achieved through hot/cold absorber measurements every $20 \mathrm{~min}$. The data were recorded using the wide-band acousto-optical spectrometer, consisting of four units with a bandwidth of $1 \mathrm{GHz}$ each, covering the $4 \mathrm{GHz}$ IF for each polarization with spectral resolution of $1 \mathrm{MHz}$.

Data were reduced using the HIPE ${ }^{1}$ and $\mathrm{CLASS}^{2}$ software packages. Spectra were calibrated using HIPE and then exported to CLASS format with the shortest possible pre-integration (typically $\sim 40 \mathrm{~s}$ ). For each scan we combined the four sub-bands in each polarization to create a $4 \mathrm{GHz}$ spectrum. From this spectrum we computed the underlying continuum using the line-free channels; we then subtracted first-order baselines from individual sub-bands. The baseline-subtracted sub-bands were again combined and the continuum level added. This results in a noiseweighted $4 \mathrm{GHz}$ spectrum for each scan. These spectra were inspected for remaining baseline instabilities, and scans with distorted baselines were omitted. For each line we inspected the co-added result in both polarizations ( $\mathrm{H}$ and $\mathrm{V}$ ) separately. The continuum level was found to agree better than $10 \%$ for each frequency. The integrated line intensities agree within $25 \%$ without significant differences of the line profiles (although the latter comparison is limited by the signal-to-noise ratio, in particular for the $557 \mathrm{GHz}$ observations). In the following we therefore use the noise-weighted average of both polarizations, which yields effective on-source integration times for the three lines are 750, 2250 , and $1300 \mathrm{~s}$ for the $\mathrm{o}-\mathrm{H}_{2} \mathrm{O}\left(1_{10}-1_{01}\right), \mathrm{p}-\mathrm{H}_{2} \mathrm{O}\left(1_{11}-0_{00}\right)$, and $\mathrm{p}-\mathrm{H}_{2} \mathrm{O}\left(2_{02}-1_{11}\right)$ lines, respectively.

Since HIFI's calibration is still preliminary, we used the theoretical predictions based on the Herschel's expected surface accuracy and the geometrical aperture size to convert the antenna temperatures to flux density (Kramer 2006). This yields $462 \mathrm{Jy} \mathrm{K}^{-1}, 467 \mathrm{Jy} \mathrm{K}^{-1}$, and $470 \mathrm{Jy} \mathrm{K}^{-1}$ at $557 \mathrm{GHz}, 988 \mathrm{GHz}$, and $1113 \mathrm{GHz}$, respectively. The final spectra are shown at a velocity resolution of 20, 10, and $25 \mathrm{~km} \mathrm{~s}^{-1}$ in Fig. 1 (top).

\section{Results}

All three water lines have been detected with high significance, demonstrating that faint (few $\mathrm{mK}$ ) broad lines can be observed with HIFI. The o- $\mathrm{H}_{2} \mathrm{O}\left(1_{10}-1_{01}\right)$ line is detected in emission and shows a double-peaked line profile. Within noise uncertainty, no emission (or absorption) is detected on the systemic velocity of M $82\left(v_{\mathrm{LSR}}=225 \mathrm{~km} \mathrm{~s}^{-1}\right)$. Both components are well fit by Gaussian profiles, with line parameters given in Table 1 . We detect a continuum level of $12.8 \mathrm{Jy}^{\text {beam }}{ }^{-1}$ at $557 \mathrm{GHz}$.

Our $1113 \mathrm{GHz}$ spectrum shows two absorption features, one centered at the systemic velocity of M 82 corresponding to the $\mathrm{p}-\mathrm{H}_{2} \mathrm{O}\left(1_{11}-0_{00}\right)$ line, and a second stronger feature blueshifted by $\sim 1.8 \mathrm{GHz}$ with respect to the systemic velocity. We identify the blue-shifted absorption feature as o- $\mathrm{H}_{2} \mathrm{O}^{+}\left(1_{11}-0_{00}\right)$

\footnotetext{
1 Herschel interactive processing environment.

2 http://WwW . iram. fr/IRAMFR/GILDAS
}

$\left(v_{\text {rest }}=1115.186 \mathrm{GHz}\right.$, Mürtz et al. 1998). Both absorption line profiles are identical within the uncertainties (see Fig. 2). The absorption profile is approximated reasonably well by a single Gaussian (see Table 1 for the line parameters). The continuum level detected at $1113 \mathrm{GHz}$ is $78 \mathrm{Jy}_{\text {beam }}{ }^{-1}$.

The $\mathrm{p}-\mathrm{H}_{2} \mathrm{O}\left(2_{02}-1_{11}\right)$ line at $988 \mathrm{GHz}$ is detected in emission. Its line profile differs from both the o- $\mathrm{H}_{2} \mathrm{O}\left(1_{10}-1_{01}\right)$ emission profile and the $\mathrm{p}-\mathrm{H}_{2} \mathrm{O}\left(1_{11}-0_{00}\right)$ absorption profiles. It shows emission between $v_{\mathrm{LSR}}=50-500 \mathrm{~km} \mathrm{~s}^{-1}$ (similar to the velocity range covered by $\mathrm{CO}$ at the same spatial resolution, see below) and is almost flat-topped for velocities between 100 and $400 \mathrm{~km} \mathrm{~s}^{-1}$. Thus the spectrum does not indicate absorption (or absence of emission) at the systemic velocity. The spectrum can be decomposed into two Gaussian profiles, with parameters given in Table 1 . The continuum flux detected at $988 \mathrm{GHz}$ is $61 \mathrm{Jy}^{\text {beam }}{ }^{-1}$.

\section{Discussion}

\subsection{The line profiles}

Given the large body of high spatial resolution observations of molecular gas tracers published for M 82, the line profile of the water lines can be compared to other data to learn more about the location and extent of the water emitting/absorbing regions in the disk. We here compare the water line profiles to the high spatial resolution $\left(3.5^{\prime \prime}\right){ }^{12} \mathrm{CO}(J=1 \rightarrow 0)$ data cube obtained by Walter et al. (2002); ${ }^{12} \mathrm{CO}$ (CO thereafter) is the best-studied tracer of the molecular gas in M 82. We first compare the CO spectra in beams synthesized to the same spatial resolutions as the HIFI beams. From the comparison of the $\mathrm{p}-\mathrm{H}_{2} \mathrm{O}\left(1_{11}-0_{00}\right)$ absorption spectrum to $\mathrm{CO}$, it is apparent that the absorption is not only detected in the pronounced absorption feature close to the systemic velocity, but also at velocities in the wings of the $\mathrm{CO}$ profile (Fig. 1 bottom). It is therefore tempting to speculate that the lack of absorption at certain velocities has a geometrical origin, i.e., that gas at these velocities is located behind the continuum. This is also supported by the shape of the $\mathrm{p}-\mathrm{H}_{2} \mathrm{O}\left(2_{02}-1_{11}\right)$ line emission profile, which shows that water is abundant in the gas phase of M 82 at all velocities where $\mathrm{CO}$ (i.e. molecular gas) is present. The very good correspondence of the $0-\mathrm{H}_{2} \mathrm{O}^{+}\left(1_{11-}\right.$ $0_{00}$ ) absorption profile near the systemic velocity suggests that the ionised water traces the same gas as is detected in the water absorption. A closer inspection of the $0-\mathrm{H}_{2} \mathrm{O}^{+}\left(1_{11}-0_{00}\right)$ profile shows, however, a lack of absorption in the red wing of the line profile (see Fig. 2). The blue wing is only partly covered by our spectrum and shows emission at the very blue edge. This could come from calibration uncertainties at the edge of the IF-band, but it is unclear whether this feature is an artifact or is real.

Our finding that the $\mathrm{p}-\mathrm{H}_{2} \mathrm{O}\left(1_{11}-0_{00}\right)$ line is observed in absorption while the $\mathrm{p}-\mathrm{H}_{2} \mathrm{O}\left(2_{02}-1_{11}\right)$ line is detected in emission can be used to obtain an estimate of the excitation temperatures of both lines. We used the dust model by Siebenmorgen $\&$ Krügel (2007) to estimate a background temperature of $18 \mathrm{~K}$ and $20 \mathrm{~K}$ at 988 and $1113 \mathrm{GHz}$, respectively. This implies $T_{\mathrm{ex}}<$ $20 \mathrm{~K}$ for $\mathrm{p}-\mathrm{H}_{2} \mathrm{O}\left(1_{11}-0_{00}\right)$ and $T_{\mathrm{ex}}>18 \mathrm{~K}$ for $\mathrm{p}-\mathrm{H}_{2} \mathrm{O}\left(2_{02}-1_{11}\right)$ (or $T_{\mathrm{ex}} \approx 19 \mathrm{~K}$ if both lines are close to LTE). Given the complexity of the water energy level diagram and the various level population channels (collisional or radiative), detailed models will be required for investigating the underlying excitation mechanisms.

Owing to the much larger beam size of the $0-\mathrm{H}_{2} \mathrm{O}\left(1_{10^{-}}\right.$ $1_{01}$ ) observations, a comparison to the other water lines is not straightforward. In comparison to $\mathrm{CO}$, however, it is apparent that the $\mathrm{o}-\mathrm{H}_{2} \mathrm{O}\left(1_{10}-1_{01}\right)$ emission arises exclusively from 

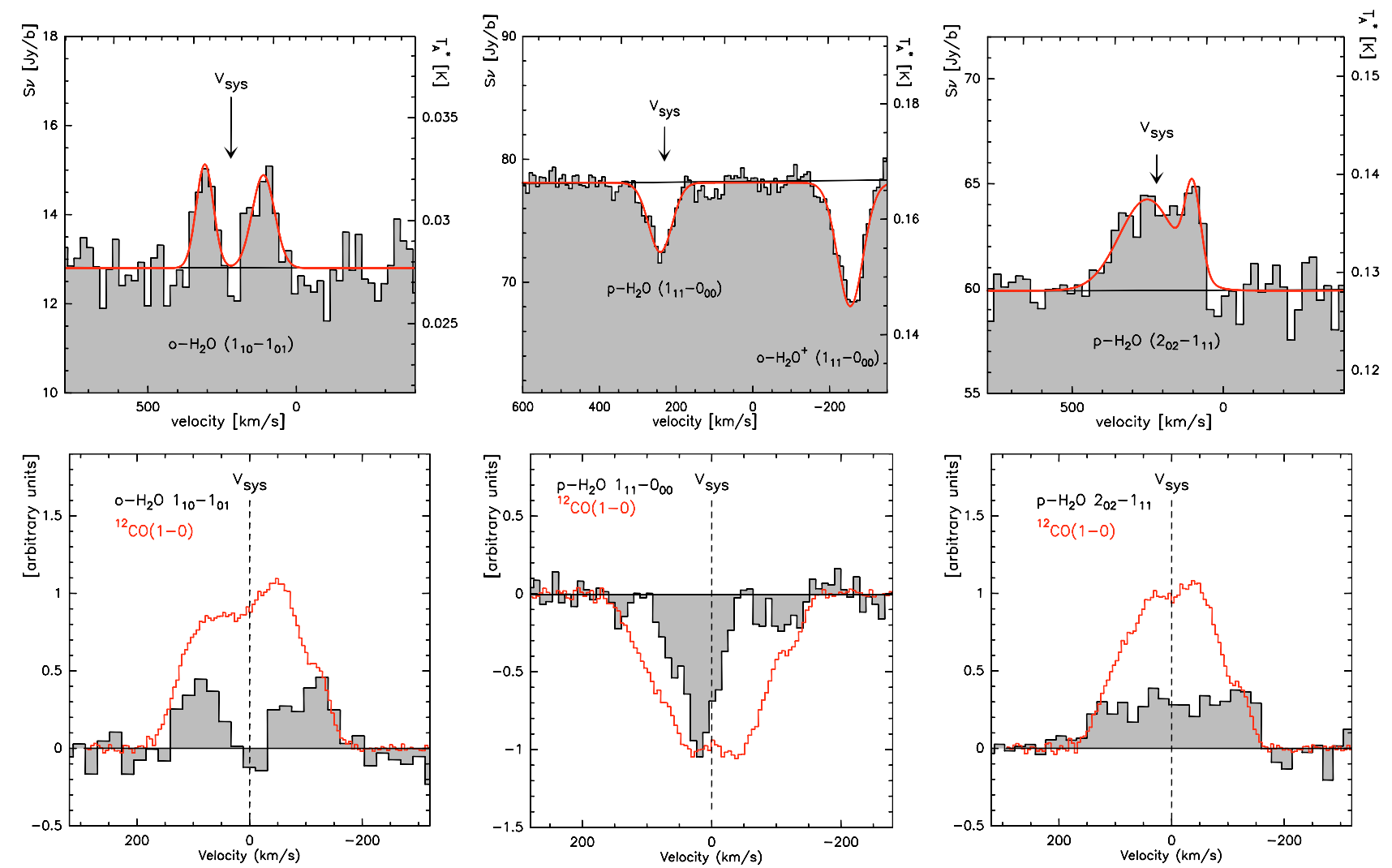

Fig. 1. Top: spectra of the $\mathrm{o}-\mathrm{H}_{2} \mathrm{O}\left(1_{10}-1_{01}\right)$, p- $\mathrm{H}_{2} \mathrm{O}\left(1_{11}-0_{00}\right)$ and $\mathrm{p}-\mathrm{H}_{2} \mathrm{O}\left(2_{02}-1_{11}\right)$ lines towards the centre of $\mathrm{M} 82$, with Gaussian-fit profiles superposed. The line parameters are given in Table 1. Bottom: water-line profiles superposed on the $\operatorname{CO}(J=1 \rightarrow 0)$ line profiles within beams synthesized with the same spatial resolution as the water data. The velocity scale is relative to the systemic velocity of M $82\left(v_{\mathrm{LSR}}=225 \mathrm{~km} \mathrm{~s}^{-1}\right)$. The intensities of the $\mathrm{CO}$ line profiles have been normalized to unity, and the water line profiles scaled to provide close matches to the $\mathrm{CO}$ profiles in the line wings. The $\mathrm{CO}$ profile in the middle panel has been inverted for better comparison to the water absorption profile.

Table 1. Line parameters derived from a Gaussian fit.

\begin{tabular}{lccrccccc}
\hline \hline Line & $\begin{array}{c}v_{\text {rest }} \\
{[\mathrm{GHz}]}\end{array}$ & $\begin{array}{c}\text { beam size } \\
{\left[{ }^{\prime \prime}\right]}\end{array}$ & $\begin{array}{r}T_{\mathrm{A}}^{*} \text { peak } \\
{[\mathrm{mK}]}\end{array}$ & $\begin{array}{c}S_{v} \text { peak } \\
{\left[\mathrm{Jy} \mathrm{beam}^{-1}\right]}\end{array}$ & $\begin{array}{c}I_{v} \\
{\left[\mathrm{Jy} \mathrm{beam}^{-1} \mathrm{~km} \mathrm{~s}^{-1}\right]}\end{array}$ & $\begin{array}{c}v_{\mathrm{LSR}} \\
{\left[\mathrm{km} \mathrm{s}^{-1}\right]}\end{array}$ & $\begin{array}{c}\mathrm{d} v \\
{\left[\mathrm{~km} \mathrm{~s}^{-1}\right]}\end{array}$ & $\begin{array}{c}S_{v} \text { cont. } \\
{\left[\mathrm{Jy} \mathrm{beam}^{-1}\right]}\end{array}$ \\
\hline $\mathrm{o}-\mathrm{H}_{2} \mathrm{O}\left(1_{10}-1_{01}\right)$ & 556.936 & 41 & $4.5 \pm 1.1$ & $2.1 \pm 0.5$ & $200 \pm 33$ & $110 \pm 8$ & $90 \pm 15$ & $12.8(540 \mu \mathrm{m})$ \\
& & & $5.0 \pm 1.1$ & $2.3 \pm 0.5$ & $170 \pm 29$ & $308 \pm 6$ & $68 \pm 11$ & \\
$\mathrm{p}-\mathrm{H}_{2} \mathrm{O}\left(2_{02}-1_{11}\right)$ & 987.927 & 23 & $8.9 \pm 2.0$ & $4.2 \pm 0.9$ & $273 \pm 90$ & $100 \pm 9$ & $61 \pm 20$ & $60.0(300 \mu \mathrm{m})$ \\
& & & $9.3 \pm 2.0$ & $4.4 \pm 0.9$ & $1005 \pm 130$ & $250 \pm 15$ & $220 \pm 27$ & \\
$\mathrm{p}-\mathrm{H}_{2} \mathrm{O}\left(1_{11}-0_{00}\right)$ & 1113.343 & 20 & $-12.0 \pm 1.6$ & $-5.6 \pm 0.7$ & $-432 \pm 28$ & $240 \pm 2$ & $72 \pm 6$ & $78.1(270 \mu \mathrm{m})$ \\
$\mathrm{o}-\mathrm{H}_{2} \mathrm{O}^{+}\left(1_{11}-0_{00}\right)$ & 1115.186 & 20 & $-21.4 \pm 1.6$ & $-10.1 \pm 0.7$ & $-836 \pm 29$ & $242 \pm 2$ & $77 \pm 5$ & \\
\hline
\end{tabular}

velocities in the line wings of the $\mathrm{CO}$ spectrum. These velocities mainly correspond to emission in the southwestern and northeastern molecular lobes that are located within the $41^{\prime \prime}$ beam (see Fig. 3 for the CO distribution compared to the HIFI beams), and yet we cannot rule out that the lack of o- $\mathrm{H}_{2} \mathrm{O}\left(1_{10}-1_{01}\right)$ emission near the systemic velocities is due to self absorption in the water line profile. Observations of the water lines within the molecular lobes will be required to obtain similar spatial coverage of all lines, thus allowing detailed analysis of different line profiles and modeling of the water excitation. These observations have been approved and will be presented in a forthcoming paper.

We retrieved sub-beam location information by comparing $\mathrm{CO}$ line profiles at the highest spatial resolution (3.5") to the p$\mathrm{H}_{2} \mathrm{O}\left(1_{11}-0_{00}\right)$ and o- $\mathrm{H}_{2} \mathrm{O}^{+}\left(1_{11}-0_{00}\right)$ line profiles. The results are displayed in Fig. 3, where we show selected $\mathrm{CO}$ spectra overlaid on the $\mathrm{p}-\mathrm{H}_{2} \mathrm{O}\left(1_{11}-0_{00}\right)$ absorption profile. We find that the $\mathrm{CO}$ line profile is in very good agreement with the water absorption profile in only a small region within the HIFI beam.
The region delineated in Fig. 3 corresponds to a small strip orthogonal to the molecular disk of M 82. The CO profiles east and west of this region show significant shifts of their line centroids compared to the peak of the water absorption due to the rotation of the molecular disk. Interestingly, the $\mathrm{CO}$ line profiles that match the shape of the main water absorption profile also show a blue wing. This CO emission corresponds to molecular gas in the outflow of M 82 (Walter et al. 2002). This shows that the water absorption in the blue line wing arises not only from the northwestern molecular lobe (which has similar velocities and is at least partly covered by the HIFI beam), but also from gas in the outflow.

\subsection{The origin of the $\mathrm{H}_{2} \mathrm{O} / \mathrm{H}_{2} \mathrm{O}^{+}$absorption}

We used the observed absorption depth to investigate whether the $\mathrm{p}-\mathrm{H}_{2} \mathrm{O}\left(1_{11}-0_{00}\right)$ and $\mathrm{o}-\mathrm{H}_{2} \mathrm{O}^{+}\left(1_{11}-0_{00}\right)$ absorption arises from small cores or from a more widespread gas phase of the ISM. 


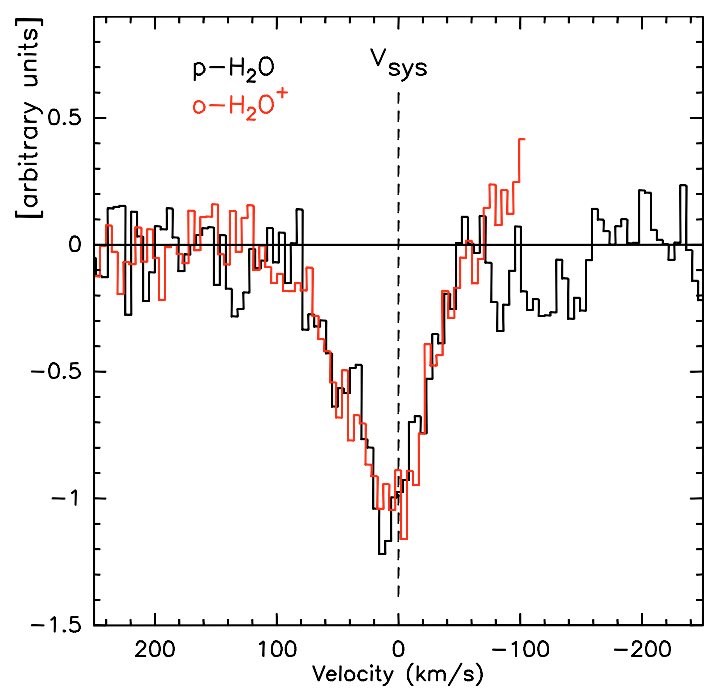

Fig. 2. Line profiles of the p- $\mathrm{H}_{2} \mathrm{O}\left(1_{11}-0_{00}\right)$ (black) and the o- $\mathrm{H}_{2} \mathrm{O}^{+}\left(1_{11}-\right.$ $0_{00}$ ) (red) lines. The velocity scale is relative to the systemic velocity of M 82. Both profiles have been normalized to a maximum absorption of unity.

For this we assume that both lines are optically thick. This implies that the absorption depth directly measures the continuum covering factor, once the continuum distribution is known. We here use the $3 \mathrm{~mm}$ continuum shown in Fig. 4 as a proxy for the submm continuum distribution. Although the continuum at this wavelength is not automatically a good indicator of the thermal dust emission, because of possible free-free contamination, the spatial similarity between the the $3 \mathrm{~mm}$ and radio continua at high spatial resolution, as well as the similarity of the $3 \mathrm{~mm}$ if smoothed to similar resolution to the published $350 \mu \mathrm{m}$ maps (Leeuw \& Robson 2009), justifies this approach. The total continuum emission of M 82 at $1113 \mathrm{GHz}$ was estimated using the dust model by Siebenmorgen \& Krügel (2007). Combining this with the observed $3 \mathrm{~mm}$ continuum distribution, we estimate $S_{1113 \mathrm{GHz}}=13.5 \mathrm{Jy}$ within the region of the water absorption. This translates into a continuum covering factor of $40 \%$ for p- $\mathrm{H}_{2} \mathrm{O}\left(1_{11}-0_{00}\right)$ and $75 \%$ for o- $\mathrm{H}_{2} \mathrm{O}^{+}\left(1_{11}-0_{00}\right)$. This approach yields predicted continuum fluxes within the HIFI beams that agree within $10 \%$ or better with the observed continuum fluxes listed in Table 1 at all three frequencies. Since the water absorption region covers $\sim 2000 \mathrm{pc}^{2}$, these numbers strongly suggest that the $\mathrm{p}-\mathrm{H}_{2} \mathrm{O}\left(1_{11}-0_{00}\right)$ and $\mathrm{o}-\mathrm{H}_{2} \mathrm{O}^{+}\left(1_{11}-0_{00}\right)$ absorption arise from a widespread gas phase of the ISM and not from dense cores (which are expected to cover a much smaller fraction of the continuum). Considering the high critical densities for collisional excitation of water (a few times $10^{7} \mathrm{~cm}^{-3}$, Faure et al. 2007), this also implies that the water excitation cannot be driven by collisions, but is most likely dominated by the IR field. This conclusion agrees with findings in other IR bright galaxies such as Arp220 and Mrk231 (e.g. Gonzalez-Alfonso et al. 2004; Gonzalez-Alfonso et al. 2010).

We can also use the absorption depth to calculated the absorbing gas column densities assuming that the absorption is not intrinsically saturated and using the continuum strength derived above (13.5 Jy). Applying the method by Menten et al. (2008) we derive $\int \tau_{\text {app }} \mathrm{d} v$ of $38.6 \mathrm{~km} \mathrm{~s}^{-1}$ and $107.2 \mathrm{~km} \mathrm{~s}^{-1}$ from the $\mathrm{p}-\mathrm{H}_{2} \mathrm{O}\left(1_{11}-0_{00}\right)$ and $\mathrm{o}-\mathrm{H}_{2} \mathrm{O}^{+}\left(1_{11}-0_{00}\right)$ absorption profiles. With the low-excitation temperature approximation, $N_{1}=$ $\frac{8 \pi v^{3}}{A_{\mathrm{ul}} c^{3}} \frac{g_{1}}{g_{u}} \int \tau_{\mathrm{app}} \mathrm{d} v$, this yields ground-level column densities of
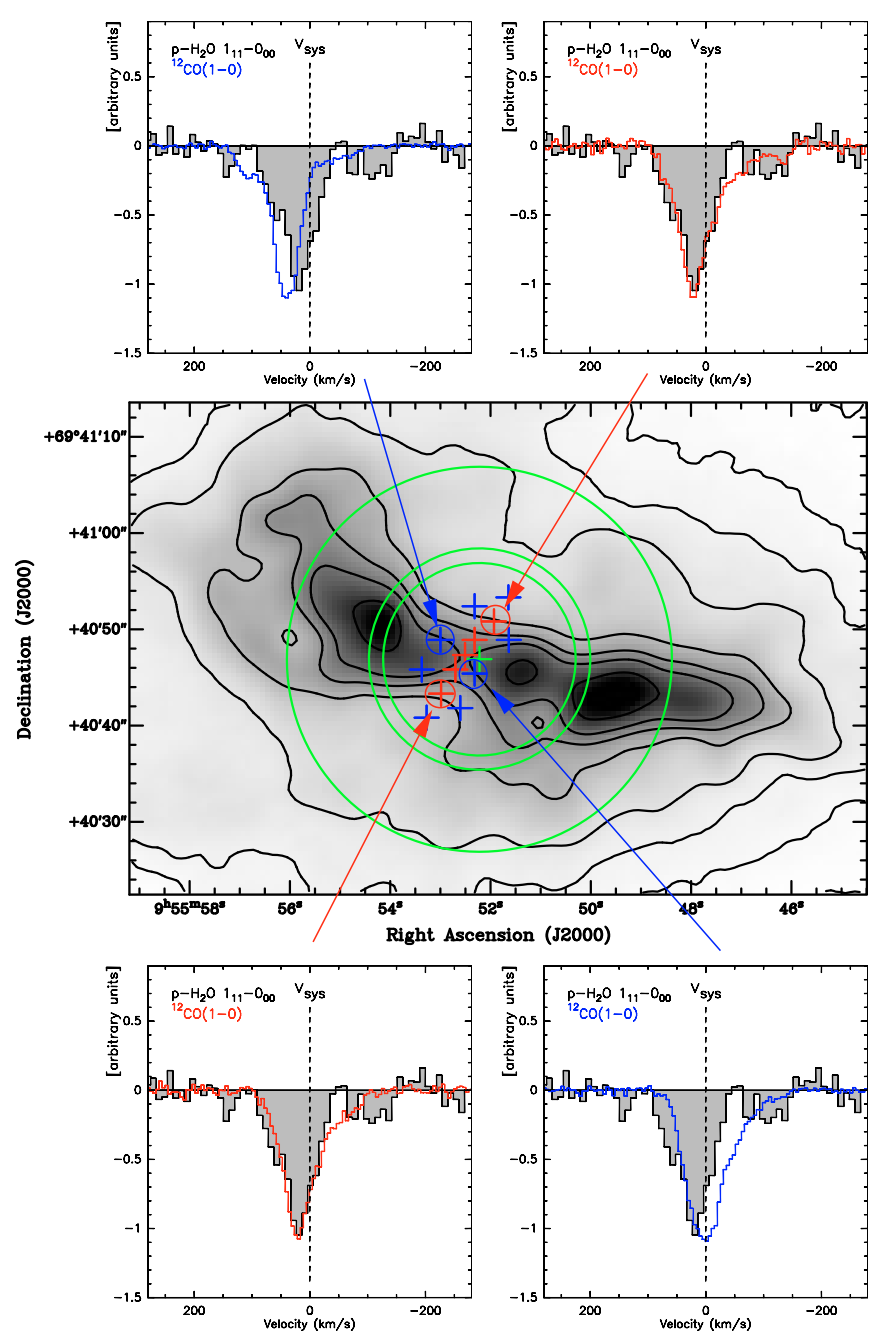

Fig. 3. HIFI beam sizes $(F W H M)$ for our observing frequencies shown on the integrated $\operatorname{CO}(J=1 \rightarrow 0)$ distribution (Walter et al. 2002) in the central region of M 82. The green cross indicates our pointing centre which corresponds to the dynamical centre of M 82. The red crosses indicate the locations where the $\mathrm{CO}$ profiles at $3.5^{\prime \prime}$ resolution match the $\mathrm{p}-\mathrm{H}_{2} \mathrm{O}\left(1_{11}-0_{00}\right)$ absorption profiles, and the blue crosses indicate locations where the $\mathrm{CO}$ profile significantly differs. The spectra to the top and bottom show example CO spectra superposed on the water absorption profiles. Their positions have also been marked by a circle for better visualization.

$N\left(\mathrm{p}-\mathrm{H}_{2} \mathrm{O} 000\right)=9.0 \times 10^{13} \mathrm{~cm}^{-2}$ and $N\left(\mathrm{o}-\mathrm{H}_{2} \mathrm{O}^{+} 000\right)=2.2 \times$ $10^{14} \mathrm{~cm}^{-2}$. For the latter, the fine structure splitting of $o-\mathrm{H}_{2} \mathrm{O}^{+}$ (see e.g. Ossenkopf etal. 2010) has been taken into account. Assuming an ortho-to-para ratio of 3:1 this results in lower limits for the total column densities of $N\left(\mathrm{H}_{2} \mathrm{O}\right) \geq 3.6 \times 10^{14} \mathrm{~cm}^{-2}$ and $N\left(\mathrm{H}_{2} \mathrm{O}^{+}\right) \geq 2.9 \times 10^{14} \mathrm{~cm}^{-2}$. By converting the measured $\mathrm{CO}$ intensities in the absorption region to proton column densities using an $X_{\mathrm{CO}}$ conversion factor as given in Walter et al. (2002), we derive limits for the fractional abundances of $\left[\mathrm{H}_{2} \mathrm{O}\right] \geq 4.0 \times 10^{-9}$ and $\left[\mathrm{H}_{2} \mathrm{O}^{+}\right] \geq 3.3 \times 10^{-9}$. These numbers are 2-3 mag lower than the water abundances derived for Arp 220 and Mrk 231 $\left(\sim 10^{-6}\right.$, Gonzalez-Alfonso et al. 2004; Gonzalez-Alfonso et al. 2010), but approach values found in molecular cloud cores, the diffuse gas in the Milky Way, and in the $z=0.89$ absorption system PKS 1830-211 (e.g. Snell et al. 2000; van der Tak et al. 2010; Menten et al. (2008). 


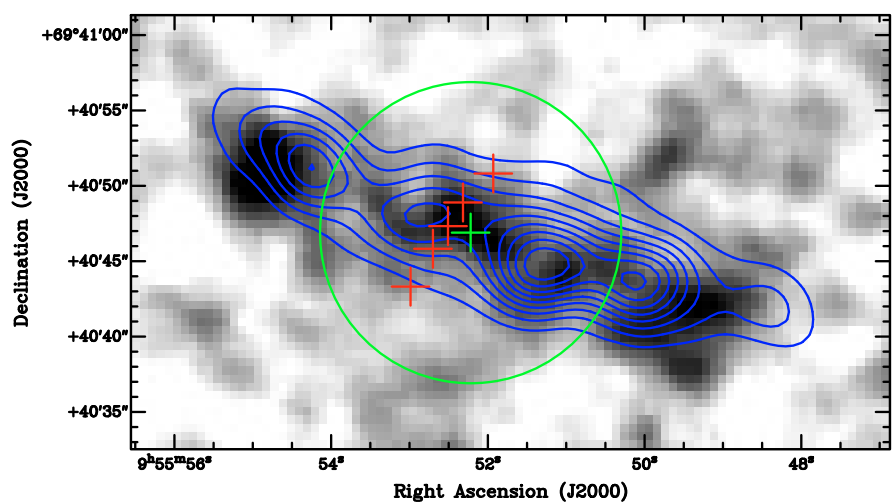

Fig. 4. 3mm continuum emission (blue contours, Weiß et al. 2001a) superposed on the integrated HCO distribution (grayscale, García-Burillo et al. 2002). The green circle indicates the $F W H M$ of the HIFI beam at the $\mathrm{p}-\mathrm{H}_{2} \mathrm{O}\left(1_{11}-0_{00}\right)$ frequency, the red crosses indicate the region of the water absorption as derived based on the $\mathrm{CO}$ line profiles (see Fig. 3).

\subsection{Comparison to other molecular gas tracers}

As shown in Fig. 3 the water absorption region is not associated

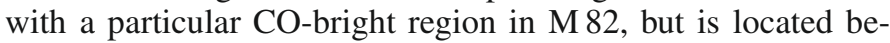
tween the northeastern molecular lobe and the central CO peak. This indicates that the water absorption is not associated with the bulk of the molecular gas in M 82 or that the underlying continuum distribution favors an absorption in regions that are not bright in CO. The latter can be ruled out by comparing the spatial distribution of the water absorption to the radio (Wills et al. 1997) and mm-continuum (Weiß et al. 2001a) as a proxy for the spatial distribution of the submm continuum (e.g. using the FIR-radio correlation). Neither tracer provides evidence that the submm continuum is in particular pronounced towards the region of the water absorption (see Fig. 4), which suggests that the small size of the water absorption is related to the ISM properties rather than to the continuum distribution.

We have further compared the spatial distribution of the dense gas as traced by HCN (Brouillet \& Schilke 1993) and $\mathrm{H}^{13} \mathrm{CO}^{+}$(García-Burillo et al. 2002) to the water absorption region. These tracers, however, closely follow the distribution of the $\mathrm{CO}$ emission, which rules out any interpretation that the water absorption occurs predominately in high-density regions.

A possible mechanism to efficiently release water from dust grains into the gas phase are shocks (e.g. Cernicharo et al. 1999). A comparison to the $\mathrm{SiO}$ distribution in M 82 (García-Burillo et al. 2001) shows that there is no indication of strong shocks in this particular region. The $\mathrm{SiO}$ observations, however, do not rule out the possibility that weaker C-type shocks could release $\mathrm{H}_{2} \mathrm{O}$ efficiently from the dust grains into the gas phase without disrupting the grains themselves. In this context it is interesting to note that the water absorption is located near the expected location of the $\mathrm{x} 2$ orbits (and their intersection with the $\mathrm{x} 1$ orbits) proposed in the context of gas motions in the stellar bar potential of M 82 (e.g. Greve et al. 2002). Shocks associated with the orbital intersection would provide a plausible mechanism for releasing water into the gas phase, and the geometry of the orbits would naturally explain why the absorption is only detected to the east of the dynamical centre of M 82 (see Fig. 2 in Greve et al. 2002).

On the other hand molecular abundances in M 82 are thought to be largely influenced by chemistry in photon-dominated regions (PDRs, see e.g. Martin et al. 2006). UV photons photodissociate water and PDR models predict that significant water gas phase abundances should only be present in well-shielded, high column density regions (see e.g. Meijerink \& Spaans 2005). This interpretation is challenged by our finding that the water absorption region in M 82 is located in a relatively low column density region within the disk of M 82. Furthermore the water absorption coincides with a peak of the spatial distribution of HCO (García-Burillo et al. 2002, see Fig. 4). HCO is a tracer of PDRs, and it is the only molecule so far imaged at high spatial resolution that shows enhanced emission in this region. The similarity of the $\mathrm{p}-\mathrm{H}_{2} \mathrm{O}\left(1_{11}-0_{00}\right)$ and $\mathrm{o}-\mathrm{H}_{2} \mathrm{O}^{+}\left(1_{11}-0_{00}\right)$ absorption profiles shows that ionizing photons are present in the absorbing medium. Therefore UV dissociation could suppress the water gas phase abundances, leading to much lower water abundances in M 82 compared to those derived in Arp 220 and Mrk 231. This is in line with studies of other molecules with low ionization potential, such as as $\mathrm{NH}_{3}$ and $\mathrm{HNCO}$, for which photo dissociation has been suggested as a main driver for the low abundances observed in M 82 (Weiß et al. 2001b; Martin et al. 2009).

Acknowledgements. HIFI has been designed and built by a consortium of institutes and university departments from across Europe, Canada, and the United States under the leadership of SRON Netherlands Institute for Space Research, Groningen, The Netherlands, and with major contributions from Germany, France, and the US. Consortium members are: Canada: CSA, U.Waterloo; France: CESR, LAB, LERMA, IRAM; Germany: KOSMA, MPIfR, MPS; Ireland: NUI Maynooth; Italy: ASI, IFSI-INAF, Osservatorio Astrofisico di Arcetri - INAF; Netherlands: SRON, TUD; Poland: CAMK, CBK; Spain: Observatorio Astronmico Nacional (IGN), Centro de Astrobiologia (CSIC-INTA). Sweden: Chalmers University of Technology - MC2, RSS \& GARD; Onsala Space Observatory; Swedish National Space Board, Stockholm University - Stockholm Observatory; Switzerland: ETH Zurich, FHNW; USA: Caltech, JPL, NHSC.

A.H., S.L. acknowledge support for this work by NASA through an award issued by JPL/Caltech. J.M.P. and J.R. have been partially supported by MCINN grant ESP2007- 65812-CO2-01. RSz acknowledges support from grant No. 203393334 from the Polish MNiSW.

\section{References}

Brouillet, N., \& Schilke, P. 1993, A\&A, 277, 381

Cernicharo, J., Pardo, J. R., Gonzalez-Alfonso, E., et al. 1999, ApJ, 520, L131

Cernicharo, J., Pardo, J. R., \& Weiß, A. 2006, ApJ, 646, 49

Combes, F., \& Wiklind, T. 1997, ApJ, 486, 79

de Graauw, Th., Helmich, F. P., Phillips, T. G., et al. 2010, A\&A, 518, L6

Faure, A., Crimier, N., Ceccarelli, C., et al. 2007, A\&A, 472, 1029

Fischer, J., Luhman, M. L., Satyapal, S., et al. 1999, Ap\&SS, 266, 91

García-Burillo, S., Martin-Pintado, J., Fuente, A., \& Neri, R. 2001, ApJ, 563, 27

García-Burillo, S., Martin-Pintado, J., Fuente, A., Usero, A., \& Neri, R. 2002, ApJ, 575, 55

Greve, A., Wills, K. A., Neininger, N., \& Pedlar, A. 2002, A\&A, 383, 56

Gonzalez-Alfonso, E., Smith, H. A., Fischer, J., \& Cernicharo, J. 2004, ApJ, 613, 247

Gonzalez-Alfonso, E., Fischer, J., Isaak, K., et al. 2010, A\&A, 518, L43

Kramer, C., Spatial Response - Contribution to the framework document of the HIFI/Herschel calibration group, HIFI/ICC/2003-30, version 1.8 (May 2006) Leeuw, L. L., \& Robson, E. 2009, AJ, 137, 517

Martín, S., Mauersberger, R., Martín-Pintado, J., Henkel, C., \& García-Burillo, S. 2006, ApJS, 164, 450

Martín, S., Martín-Pintado, J., \& Mauersberger, R. 2009, ApJ, 694, 610

Meijerink, R., \& Spanns, M. 2005, A\&A, 436, 397

Menten, K., Güsten, R., Leurini, S., et al. 2008, A\&A, 492, 725

Mürtz, P., Zink, L. R., Evenson, K. M., \& Brown, J. M. 1998, JChPh, 109.9744 Ossenkopf, V., Müller, H. S. P., Lis, D. C., et al. 2010, A\&A, 518, L111

Sakai, S., \& Madore, B. F. 1999, ApJ, 526, 599

Siebenmorgen, R., \& Krügel, E. 2007, A\&A, 461, 445

Snell, R. L., Howe, J. E., Ashby, M. L. N., et al. 2000, ApJ, 539, 101

van der Tak, F. F. S., Marseille, M. G., Herpin, F., et al. 2010, A\&A, 518, L107

Van der Werf, P. P., Isaak, K. G., Meijerink, R., et al. 2010, A\&A, 518, L42

Walter, F., Weiß, A., \& Scoville, N. Z. 2002, ApJ, 580, 21

Wills, K. A., Pedlar, A., Muxlow, T. W. B., \& Wilkinson, P. N. 1997, MNRAS, 291,517

Weiß, A., Neininger, N., Hüttemeister, S., \& Klein, U. 2001a, A\&A, 365, 571

Weiß, A., Neininger, N., Henkel, C., Stutzki, J., \& Klein, U. 2001b, ApJ, 554, 143 